\title{
Das «operative» Konzept der Institution
}

\author{
Thomas und Elisabeth von Salis (Zollikon)
}

Zusammenfassung: Die Institution und die Gruppe werden in dieser Arbeit im Sinne der "operativen Gruppenkonzepte», fussend auf Pichon-Rivière und seinen Nachfolgern (Bléger, Bauleo und andere argentinische Autoren), definiert. Anhand klinischer Beispiele von Interventionen in psychiatrisch-psychotherapeutischen und pädagogischen Einrichtungen illustrieren wir die Arbeitsweise und etwas von der Wirkung, soweit dies ohne begleitenden forschungstechnischen Apparat möglich ist. Dabei geht es um eine Theorie und Praxis, die die Gruppe zum Gegenstand der psychoanalytischen klinischen Forschung, Therapie und Didaktik macht. Zentral und für die Methode kennzeichnend ist das Gewicht, das aufdas Setting und dessen verschiedene konstituierenden Elemente, nämlich die Gruppenaufgabe, die Koordination und Beobachtung/Interpretation, sowie die in der psychoanalytischen Praxis geläufigen Faktoren der Präsenz der Mitglieder in geregelten räumlich-zeitlichen und ökonomischen und Rollen-Verhältnissen gelegt wird. Wir berichten im Folgenden über einige rezente Erfahrungen mit Mitarbeiter-Teams in klinischen und pädagogischen Einrichtungen.

Schlüsselwörter: Psychoanalyse, operative Gruppe, Institution, Ideologie, Praxis

\section{Zum Konzept}

Das operative Konzept der Institution befindet sich in einem gutnachbarlichen Verhältnis zu den Konzepten der Institutionsanalytiker Lourau und Lapassade (s. weiter unten). Die Institution wird Bauleo zufolge als ein intergruppaler Raum aufgefasst. ${ }^{1}$ Die Gruppen und Untergruppen einer Einrichtung oder Organisation $^{2}$ (eines Etablissements) wie einer Schule, einer politischen Partei oder einer psychoanalytischen Gesellschaft sind von institutionellen Momenten geprägt, von Machtfaktoren, Ideologien und Implikationen. Die operativen Konzepte, wie wir sie vor allem von Armando Bauleo (2013) gelernt haben, gehen auf Pichon-Rivière und José Bléger in Argentinien zurück. Wir befinden uns in regem Austausch mit italienischen und spanischen Kolleg*innen, die seinerzeit in 
den 1980er-Jahren etwa gleichzeitig mit uns die Anwesenheit der exilierten argentinischen Kolleg*innen in Europa nutzen konnten, um sich mit den operativen Gruppenkonzepten vertraut zu machen. Wir arbeiten mit diesen Konzepten in der Regel in der Kleingruppe. Die Grossgruppe, wie zum Beispiel die Vollversammlung, ist das von den Institutionsanalytikern bevorzugte Setting, und sie wird auch von den Gruppenanalytikern gepflegt, viel weniger von Analytikern, die mit den operativen Konzepten arbeiten.

Diese Konzepte rücken den Begriff der Aufgabe als organisierendes Element ins Zentrum. Die Gruppe wird definiert als eine Anzahl von Personen, die anhand einer gemeinsamen Aufgabe ein Ziel verfolgt und dabei zu einer Gruppenstruktur gelangt. Die Beziehungen unter den Teilnehmern stehen in ihrer Qualität in Zusammenhang mit der Aufgabenbezogenheit der Gruppenarbeit. Ein Gefühl der Zugehörigkeit stellt sich mit zunehmender Erfahrung im Gruppenprozess ein. Der Gruppenprozess wird begleitet und beeinflusst von der Koordination und Beobachtung, die in der Regel von zwei dazu qualifizierten Personen durchgeführt wird. Ihre Aufgabe ist verschieden von derjenigen der Gruppenmitglieder und besteht in der Beobachtung und Deutung dessen, was in der Gruppe vor sich geht, stets im Hinblick auf das Verhältnis der Gruppe zu ihrer Aufgabe. Es werden also nicht die Äusserungen oder das Verhalten der einzelnen Mitglieder gedeutet, sondern der Umgang der Mitglieder mit der Aufgabe. Das besondere Augenmerk der Koordinations-Equipe ${ }^{3}$ richtet sich auf latente Aspekte der Aufgabe, die der Gruppe in geeigneter Form und mit adäquatem Timing gedeutet werden.

\section{Zwei Einrichtungen in Frankreich}

Der kinderpsychiatrische Leiter eines psychologisch-pädagogischen Dienstes engagierte uns für seinen Dienst in einer französischen Stadt, um den Mitarbeitern das operative Gruppenkonzept nahezubringen. Er versprach sich davon eine Verbesserung der Zusammenarbeit in der Belegschaft, die aus psychologisch-therapeutischen und pädagogischen Fachpersonen bestand: Psychoanalytiker*innen, Logopäd*innen, psychomotorischen Therapeut*innen, Sekretärinnen, einer administrativen Direktorin (Soziologin), einer Pädiaterin und dem kinderpsychiatrischen Leiter. Zwei Monate nachdem wir die Arbeit begonnen hatten, holte uns die administrative Direktorin, die an den Gruppensitzungen teilnahm, an eine zweite, gleichartig strukturierte Einrichtung in der Nachbarstadt, die auch unter ihrer administrativen Leitung stand. Dieser Dienst hatte eine Erwachsenenpsychiaterin als ärztliche Leiterin. Die beiden Leitungspersonen litten unter den schwelenden Konflikten in der Einrichtung. Die Administratorin emp- 
fahl nun aufgrund der Erfahrungen in der anderen von ihr verwalteten Institution dieser Belegschaft, eine Einführung ins operative Gruppenkonzept durchzuführen. Schon seit mehr als einem Jahr hatte die Belegschaft eine Supervision gefordert, war sich dabei aber über die Realisierung nie einig geworden.

Bei Supervisionsaufträgen sollte in einem Erstinterview geklärt werden, um was für ein Team es geht. Können wir, wie Armando Bauleo fragt (1997, S. 89),

seine Dynamik erkennen, die in ihm spielenden Beziehungen, den Code, den die Mitarbeiter geschaffen haben, die Art und Weise, wie sie mit den Hierarchien umgehen, und die Konflikte, die im Spiel sind?

Diese Vorarbeit und Einschätzungen wurden in gewissem Masse von den Leitungspersonen schon geleistet.

Den Nutzen der Gruppenarbeit mit Teams in therapeutischen oder pädagogischen Einrichtungen konnten wir in früheren Interventionen unter anderem darin erkennen, dass das Zeitmanagement besser gelingt und damit der Druck der Pendenzen von den Teilnehmer*innen allmählich als weniger unerträglich erlebt wird. Diesen Effekt beobachteten wir in der Schweiz bei zwei sehr verschiedenen Gelegenheiten und nun auch in den beiden Einrichtungen in Frankreich. Wir führen dies darauf zurück, dass man in der operativen Gruppe lernt, vermehrt auf die Gruppenaufgabe zu achten und damit die Aufmerksamkeit von irgendeiner emotionalen Fixierung (z. Bsp. auf die zu grosse Warteliste) auf den Umgang mit der Aufgabe zu verschieben. Damit wird weniger Gewicht auf die Personen und Beziehungen gelegt als auf die gemeinsame Arbeit und die jeweils gegebene Aufgabe. Der Aufgabenbegriff wird im Laufe des Gruppenprozesses klarer, konkreter und auch auf einem neuen Abstraktionsniveau definiert.

Die ideologischen Implikationen, die mit der «Aufgabe» einhergehen, können von der Gruppe allmählich einer sachgemässen Kritik unterzogen werden. Damit verändert sich freilich das Denken; wir würden mit Pichon-Rivière sagen, das Denken werde überhaupt erst möglich. Das Unterscheidungsvermögen schult sich anhand der Aufgabenbezogenheit, die sich in der Gruppe allmählich einstellt. Die emotionalen und ideologisch unterlegten Momente, die im Umgang mit der Gruppenaufgabe eine Rolle spielen, werden untersucht, oft von der koordinierenden Equipe gedeutet, damit deren Zusammenhänge mit dem Kontext, in dem die Gruppenarbeit geschieht, klarer werden. 
Wir haben die Erfahrung gemacht, dass verschiedene Teams, die wir als operative Gruppe koordinierten, sehr unterschiedlich «emotional aufgeladen» funktionieren, und zwar im Setting zuweilen völlig anders als im Alltag des Betriebs. Die Abwehr, die sich gegen Veränderung und Ängste richtet, die mit neuer Information und ungewohnten Situationen einhergehen, kann sich einmal in einer lauten Emotionalität, ein anderes Mal in einer stillen Gleichgültigkeit äussern. Dabei überrascht uns immer wieder, dass Gruppenprozesse zu mehr Aufgabenbezogenheit führen, obwohl wir und die Gruppen fast nur mit der Abwehr zu kämpfen haben.

Supervisions-Anfragen hatten wir auch immer wieder von pädagogischen Einzelpersonen oder Institutionen erhalten, und hierbei erwies sich die Arbeit jeweils als leichter durchführbar, wenn ein psychoanalytisches Grundverständnis bereits vorhanden war. Andernfalls wären zwei ideologische Widerstände zu bearbeiten: gegen die Psychoanalyse und gegen «die Gruppen».

\section{Die Maturitätsschule}

Wir berichten im Folgenden über eine pädagogische Institution, eine Maturitätsschule für Erwachsene in Deutschland, deren Schüler*innen und Lehrpersonen, die sich für die operativen Gruppen interessierten, mit uns und zwei anderen Kolleg*innen ${ }^{4}$ der AGOG (Arbeitsgemeinschaft für operative Gruppen) an zwei Intensiv-Wochenenden arbeiteten, um mit den operativen Konzepten etwas potentiell Hilfreiches für ihre Schule kennen zu lernen.

Die Teilnehmer revidierten ihre bis anhin so gut wie unhinterfragte «Freiheitsideologie», der zufolge die Schüler nach Belieben den Unterricht in der Klasse besuchen oder ihm fernbleiben können. Sie überlegten sich in den koordinierten und beobachteten operativen Gruppen, ob sie ihren bisherigen traditionellen Frontalunterricht in den Klassen gänzlich neugestalten möchten, unter Anwendung der operativen Konzepte. Im Laufe der Gruppensitzungen beklagten sich die Teilnehmer*innen darüber, dass der Unterricht immer wieder nicht stattfinden könne, da keine Schüler anwesend seien. Sie erkannten dabei die Bedeutung des Settings, also eines bestimmten Raumes an einem festgelegten Ort und zu einer bestimmten Zeit, wo die Gruppenmitglieder sich treffen. Bei der Gegenüberstellung der Freiheit, dem Unterricht fernzubleiben, mit dem Zwang, sich an ein Setting zu halten, entstand allmählich eine Sensibilität für die Dialektik des Rahmens. Die Schüler und Lehrer in den operativen Gruppen stellten fest, dass sie sich bisher erstaunlich wenig gekannt hatten. Sie konnten in der durch das Gruppensetting gebotenen unmittelbaren Präsenz und Interaktion ein aktives Lernen beginnen, 
das mit einem Interesse aneinander und einer Verbundenheit untereinander einherging und gewisse bisher feststehende Regeln in Frage stellte.

Wir hofften natürlich, dass diesen «Intensivos» ${ }^{5}$ noch eine Fortsetzung mit einem langfristigen Setting regelmässig stattfindender Gruppensitzungen folgen könnte. Dafür fehlten bis zum jetzigen Zeitpunkt jedoch noch die Finanzierungsmöglichkeiten.

\section{Eine psychiatrische Klinik}

Ein Beispiel, das wir in einem Kurs für Teamsupervision vorgebracht haben, stammt aus unserer Arbeit in einer psychiatrischen Klinik. Eine Gruppe aus der Belegschaft des grossen Spitals hatte in der dritten Sitzung eine Information von einer leitenden Angestellten bekommen, die unserer Ansicht nach nicht so recht zu der uns aufgetragenen Arbeit passen mochte. Ich (TvS) machte als Koordinator den Fehler, dass ich dazu eine Bemerkung machte, die die Informatorin in Rage versetzte. Einige jüngere Mitglieder der Gruppe nahmen die Referentin daraufhin in Schutz. Die emotionale Bewegung wogte schliesslich derart auf, dass sich der Auftraggeber unserer Arbeit genötigt fühlte, die begonnene Gruppenarbeit abzubrechen und eine Sondersitzung einzuberufen. Damit geriet das ganze Gefüge, insbesondere das von uns eingeführte und anfänglich gelungene Setting, durcheinander.

In diesem grossen Betrieb herrschte hinter der Fassade eines liberalen und nicht autoritären Führungsstils die latente Ideologie, die wir mit Robi Friedman als «Soldatenmatrix» bezeichnen würden (2018, S. 7 ff.), eine ideologische Einstellung kriegerischer Natur, die Friedman selbst als Kriegsteilnehmer und als klinischer Psychologe und Gruppenanalytiker bei Patienten und vor allem bei Grossgruppen kennenlernte. Man kann sie auch mit dem gängigen Spruch «wer nicht für uns ist, ist gegen uns» zusammenfassen. Wir waren also in jener Gruppensituation nicht mehr die Koordinations-Equipe, sondern in einer fulminanten Übertragung zu feindlichen Eindringlingen geworden. Die Person, die uns bei unserem Eintreffen in der Klinik in Empfang genommen hatte, hatte dies schon vor Beginn der Sitzungen unfreiwillig bestätigt, indem sie uns mit einer mitleidigen Bemerkung auf die Schwere unserer Aufgabe verwies.

Widerstände gegen die Psychoanalyse und gegen «Gruppen» machen uns stets, wenn auch in unterschiedlicher Ausprägung und unter den allerverschiedensten Gestalten zu schaffen, wie sie der Soziologe und Erziehungswissenschaftler Erich O. Graf (2019, S. 204) beschreibt: 
Seit jeher sind Positionen, die antiautoritär sein könnten, kritisiert, verhöhnt, verspottet, lächerlich gemacht, bekämpft, verfolgt und ausgestossen worden; dies gilt für den Marxismus so gut wie für die Psychoanalyse. Institutionalisierung und Autoritarismus hängen eng zusammen. Es kann keine antiautoritäre Institution geben, auch keine "Gegeninstitution»; das einzige was geht, ist die ständige Bewegung, die Bereitschaft, Errungenes, Erkämpftes, Erworbenes unter den sich verändernden Bedingungen neu zu denken, umzuorganisieren, sich selbst zu verändern. Das bedeutet unangenehmerweise auch, dass wir zwar ständig Institutionen aufbauen, weil sie als Teile unserer Kultur unverzichtbar sind, während wir sie gleichzeitig mit jedem Lernschritt verändern und manchmal auch wieder zerstören müssen.

Die Institutionen im Kopf werden oft mit dem Begriff der Transversalität ${ }^{6}$ belegt: Wir können in den Gruppen, die wir koordinieren, manchmal deutlich wahrnehmen, wie der gruppale Diskurs von Ideologien durchzogen ist, die von gesellschaftlichen Institutionen abstammen. Es wird damit auf eine Autoritätverwiesen, was schon als Hinweis auf institutionelle Zusammenhänge gelten kann.

Sowohl im Erziehungswesen als auch in der Psychiatrie gibt es Betriebe, in denen

die Aufgabe der Teamsupervision insofern in Frage steht, als die Aufgabe des Teams in der Institution unklar ist - die manifeste Aufgabe des Teams, die Patienten (oder sonst wie Schutzbefohlenen) $z u$ betreuen und einen geordneten Betrieb der Institution zu gewährleisten, hinter die latente zurück tritt, die gerade nicht dem Wohl der Klienten dient. Die Institution hatte in solchen Fällen vielmehr von gesellschaftlichen Kräften den Auftrag erhalten, die Klienten aus dem gesellschaftlichen Zusammenhang herauszuhalten, "aufzubewahren», zu isolieren und zu sequestrieren. (T. von Salis, 2019, S. 187)

Eine solche Auffassung der institutionellen Aufgabe, die freilich nicht explizit so formuliert wird, macht es dem Team und den Koordinatoren schwer, sich in einem operativen Gruppenprozess der Erarbeitung der Gruppenaufgabe zu widmen. Die dazu erforderliche Flexibilität in der Rollenverteilung - wie sie im 
Folgenden illustriert wird - ist nur gegen die grössten Widerstände zu gewinnen. Und wenn sie gewonnen würde, wäre das Team insofern mit dem institutionellen Konflikt belastet, als es sich im Widerspruch zur latenten institutionellen Aufgabe befindet, zu der das Team sich einer Verwahrungspolitik konform verhalten müsste. Eine solche Verwahrungspolitik entspricht eher dem Paradigma einer «Soldatenmatrix» als demjenigen einer freiheitlichen demokratischen Gesellschaftsordnung.

Die Rollenverteilung im Setting verlangt jedoch, dass die Koordination sich nicht an der Arbeit an der Gruppenaufgabe beteiligt. Die Rollen der Gruppenteilnehmer werden sich im Laufe des operativen Gruppenprozesses innerhalb und ausserhalb der Gruppe verändern, wobei sie zugleich die Rolle als Gruppenmitglied beibehalten (Die Gruppenmitgliederrolle gehört wie die Rollen der koordinierenden Equipe zum Setting).

Der im Beispiel der psychiatrischen Klinik dargestellte Fehler des Koordinators, der eine kritische Bemerkung zum Inhalt der Information gemacht hatte, bestand darin, dass er - im Gegensatz zu seiner Rolle - es nicht den Gruppenteilnehmern überliess, die gegebene Information zu bearbeiten. Die Gruppenteilnehmer*innen einer operativen Gruppe äussern im Anschluss an die Information ihre Assoziationen und Meinungen. Die Annahme, dass die Einfälle der Gruppe, die auf die Information folgen, inhaltlich auf die Information bezogen sind, auch entgegen dem Augenschein, gilt hier ebenso wie in der klinischen Psychoanalyse. (Die Einfälle der Analysand*innen betrachtet der Analytiker / die Analytikerin stets unter dem Gesichtspunkt der Motivation, die die Analysand*innen zur Analyse gebracht haben). Die Koordinator*innen und Beobachter*innen deuten die Einfälle im Hinblick auf die Gruppenaufgabe. Die Gruppe arbeitet, unterstützt durch die Deutungsarbeit, mit ihren eigenen Möglichkeiten und in ihrem eigenen Tempo an ihrer Aufgabe. Dass der Koordinator die Information nicht adäquat fand, hätte er vorerst nicht sagen sollen. Sein Eindruck war allerdings ein Hinweis darauf, wie die Aufgabe von der Informantin verstanden wurde. Der Koordinator sollte zuwarten, bis er anhand der Assoziationen im Gruppengespräch einen Zusammenhang mit der institutionellen Annahme herstellen kann. Erst jetzt deutet er, wie sich die institutionelle Annahme und ihre Ideologie anhand der so gegebenen Information bemerkbar gemacht haben. Im nachhinein könnte der Aufruhr nach der verfehlten Intervention damit zu tun haben, dass für das grosse Spital trotz aller Bemühungen um Modernität ein Stück weit der Aufbewahrungs-Auftrag galt und die therapeutischen Ansätze zu kurz kamen. Die 
betreffende Information hatte sich tatsächlich auf juristische Rechtfertigung von Zwangsmassnahmen bezogen.

Wir machen einen Unterschied zwischen $\mathrm{zu}$ deutenden Institutionen und der Einrichtung, oft auch Institution genannt, in welcher gearbeitet wird. Institutionen sind mehr oder weniger gefestigte «allgemeingültige» Annahmen und machtgestützte Regelsysteme. In der Gruppenarbeit können bei der Arbeit an der Aufgabe allmählich solche ideologisch geprägten Annahmen aufgegriffen, in Frage gestellt und bearbeitet werden. So entsteht ein vertieftes und dem Kontext, in welchem gearbeitet wird, entsprechendes Aufgabenverständnis.

Dieses Element, die Gruppenaufgabe, kann von der Gruppe nicht von Anfang an gemeinsam und im Einverständnis aller Beteiligten festgesetzt werden, weil noch kein Prozess stattgefunden hat, in dessen Verlauf die Aufgabe allmählich geklärt und von der ganzen Gruppe akzeptiert wird.

\section{Ein anfängliches Fehlverständnis}

Ein Beispiel für die häufige Anfangsschwierigkeit entnehmen wir dem im Buch Erfahrungen mit Gruppen beschriebenen Erfahrungsmaterial (Graf \& E. von Salis, 2003, S. $115 \mathrm{ff}$.).

Im ersten Jahr der Gruppenarbeit mit einem Team referierte ein Gruppenmitglied, für die Equipe völlig überraschend, anstelle eines persönlichen Berichts über die «Erfahrungen in den ersten Gruppensitzungen» wie es vereinbart war, einen Text («Umgang mit dem Tod in den Institutionen»), den sie aus einem ganz anderen Zusammenhang ihrer Fort- und Weiterbildung ausgewählt hatte. Hier zeigte sich einerseits die Angst vor Verlust und Veränderung, die nicht persönlich und auf die gegenwärtige Erfahrung bezogen formuliert wurde. Andererseits entsprach es dem eingeübten Umgang mit Fortbildung, die aus Vorträgen von externen Fachleuten bestand. Man kopierte die externen Dozenten statt sich auf die Aufgabe einzulassen.

Im zweiten Jahr wurde - neu für eine Gruppensitzung - ein Tonbandgerät eingeschaltet. Bei der Klärung wie es dazu gekommen sei, konnte die Unterscheidung

zwischen der Gruppenarbeit im Setting und der sonstigen Fortbildung gemacht werden.

Im dritten Jahr zeigten sich noch immer die stereotypen Rollenmuster und die Schwierigkeiten, die Verantwortung für die Information zu übernehmen. Schon zweimal war es vorgekommen, dass niemand im Voraus bereit war zu referieren und dann jemand es spontan übernommen hatte, eine Information zu bringen. Sie nannten das «Feuerwehrübung» und wollten so nicht weiterfahren. 
«Feuerwehrübungen» nannten sie auch die notfallmässigen Interventionen in Familien. Sie imitierten überdies die Chefin, die zwar als Teilnehmerin dabei war, aber bisher nicht referiert hatte.

Nun aber entschloss sich die Chefin, auch einmal die Information zu präsentieren. Dabei merkten die Mitarbeiter*innen, dass die Einrichtung nicht dasselbe war wie unser Setting. In der Gruppe galt nicht dieselbe Rollenverteilung wie im Betrieb. Sie hatten den Unterschied zwischen Innerhalb und Ausserhalb des Settings bis dahin noch nicht gemacht.

Nachdem nun aber die Chefin referiert hatte, wurde der Rollenwechsel auch den übrigen Teilnehmer*innen möglich, und das Referieren war kein Problem mehr. Hier sehen wir ein institutionelles Moment am Werk. Wenn die Chefin ihre Rolle wechseln konnte, konnten es auch die Team-Mitglieder. Man verabschiedete sich von einer ideologischen Position, um operativ in der Gruppe zu arbeiten. Man kopierte nicht mehr die Chefin, sondern konnte selbst denken. Damit veränderte sich eine ideologische Grundannahme, die besagte, dass hierarchische Rollen in einem Betrieb nicht in Frage gestellt werden dürfen.

Wir müssen oft gut hinhören, um einerseits die Widerstände und andererseits die Fortschritte (oder Rückschritte) in der Aufgabenbezogenheit und dem Kommunikationsstil und eben auch Wechsel in der Rollenverteilung im Sinne einer Flexibilisierung, wahrnehmen zu können.

Nach Freud und der Inschrift auf seinem Denkmal in Wien ist «die Stimme der Vernunft [...] leise, aber sie ruht nicht, ehe sie sich Gehör verschafft hat. ${ }^{7}$ Der Effekt, der im Lauf des Gruppenprozesses erkennbar wird, ist die «Vernunft» im Sinne der Aufgabenbezogenheit. Emotional äussert sich dieser Faktor als Zugehörigkeitsgefühl. Wir schliessen aus der Beobachtung vermehrter Aufgabenbezogenheit und des verstärkten Zugehörigkeitsgefühls auf eine Gruppenstruktur, die sich gebildet hat. Die Beziehungen unter den Mitgliedern sind im Laufe der zunehmenden Gemeinsamkeit im Auffassen der Gruppenaufgabe flexibler und operativ geworden.

Die Bewusstseins- und Gefühlsmomente, von denen hier die Rede ist, unterscheiden sich radikal von denjenigen in Grundannahmen-Gruppen, wie sie Bion beschreibt. Bion unterschied die arbeitende Gruppe von derjenigen, die sich nach Grundannahmen richtet. Letztere haben etwas Stereotypes, Phantasiegesteuertes; die Führungsrolle ist dabei oft auf eine Person fixiert worden, sei es in einer positiven (Beispiel: «Guru») oder negativen Führungsrolle (zum Beispiel als «Patient der Gruppe»). Die Aufgabe kann als etwas Wahnhaftes beschrieben werden (Bion (2001, Abschnitte zu den drei Grundannahmen: Flucht-Kampf-Gruppe, 
Abhängigkeitsgruppe, Paarbildungsgruppe). Da Bion nicht mit dem operativen Konzept arbeitete, das damals gerade allmählich in Buenos Aires entwickelt wurde, verliefen die von ihm beobachteten Prozesse anders. Seine Beobachtungen betrafen Gruppenzustände, die wir kaum je in so krasser Form zu sehen bekommen, da unsere an der Beziehung zur Gruppenaufgabe orientierte Deutungstechnik gestattet, die auftauchenden Ängste auf einem annähernd optimalen Niveau zu halten, sodass auch der Abwehraufwand sich in Grenzen hält.

Bis hierher beschränkten wir uns auf klinische Beobachtungen. Die Arbeit im Setting kann man als semi-experimentelle Forschungsarbeit auffassen; dabei ist es seit Anbeginn ein Problem, wie man das, was in der Gruppe geschieht, notiert und dokumentiert. Wir haben das handschriftliche Notieren dessen, was in der Gruppe geschieht, dem Beobachter/der Beobachterin zugeteilt. Die Gruppe bekommt gegen Ende der jeweiligen Sitzungen eine Rückmeldung über das, was beobachtet wurde. Die Gegenübertragungsmomente werden dabei, so gut es geht, als Erkenntnisquelle benützt.

Von einer ganz anderen Warte her könnte man mittels Fragebogen Daten bei den Gruppenteilnehmern erheben, um zu verfolgen, wie sich die subjektive Verfassung bei den Teilnehmer*innen im Verlauf des Gruppenprozesses verändert. Hier stehen wir ganz am Anfang und können noch keine Resultate präsentieren.

\section{Literatur}

Bauleo A. (1997). Die institutionelle Supervision. Sondernummer «Zur Theorie und Praxis der operativen Gruppe». Journal für Psychoanalyse, 87-92.

Bauleo, A. (2013). Ideologie, Familie und Gruppe. In von Salis (Hrsg.), Beiträge zur Analyse von Gruppen und Institutionen Bd. I. Wien: LIT Verlag (S. 184-190). Bion, W. (2001). Erfahrungen in Gruppen und andere Schriften. Stuttgart: Klett-Cotta. Friedmann, R. (2018). Die Soldatenmatrix und andere psychoanalytische Zugänge zur Beziehung von Individuum und Gruppe. Giessen: Psychosozial.

Graf, E. \& von Salis, E. (2003). Erfahrungen mit Gruppen. Theorie, Technik und Anwendungen der operativen Gruppen. Zürich: Seismo.

Lapassade, G. (1972). Gruppen, Organisationen, Institutionen. Stuttgart: Klett. von Salis, T. (2019). Das Lernen und die Gruppe. Arbeiten zum Thema der operativen Gruppen. Beiträge zur Analyse von Gruppen und Institutionen Bd. II. Wien: LIT Verlag. 


\section{Anmerkungen}

1 Mündliche Mitteilung Bauleos anlässlich zahlreicher Diskussionen.

2 Im Sinne von Georges Lapassade (1972, S. 84).

3 Gruppenanalytiker sprechen von Leitung und Ko-leitung, während wir unsere Rolle anders, eben im Sinne der Koordination und Beobachtung verstehen.

$4 \quad$ Christine und Thomas Greusing.

5 Mit «Intensivo» bezeichnete Bauleo Gruppenseminarien, die auf wenige Tage komprimiert sind und an denen mehrere Sitzungen pro Tag stattfinden.

6 Le terme «transversalité» est rapidement repris dans «une perspective psychosociologique trop réductionniste» (selon Guattari) par Georges Lapassade et René Lourau (avant tout dans son article «Le syndicalisme: de l'institution à la bureaucratie» puis dans sa thèse d'État L'analyse institutionnelle.) Pour ce dernier, la transversalité désigne la «résultante» (dissimulée grâce à l'idéologie individualiste et universaliste) de la pluralité des appartenances et références segmentaires. L'objet d'une analyse institutionnelle des groupes est de mettre à jour la transversalité comme institution.«https:// fr.wikipedia.org/wiki/Transversalité_(sciences_humaines_et_sociales) abgerufen 18.02.2020. Auf Deutsch: Der Begriff «Transversalität» wird von Georges Lapassade und René Lourau (zunächst in seinem Artikel «Le syndicalisme: de l'institution à la bureaucratie » und dann in seiner Dissertation L'analyse institutionnelle) in «einer (nach Guattari) zu reduktionistischen psychosoziologischen Perspektive» aufgegriffen. Für letztere bezieht sich die Transversalität auf das «Resultat (das dank der individualistischen und universalistischen Ideologie verborgen ist) der Pluralität der segmentalen Zugehörigkeiten und Bezüge. Der Zweck einer institutionellen Analyse von Gruppen ist es, die Transversalität als Institution aufzudecken». 7 https://gutezitate.com/zitat/187920.

\section{Angaben zur Autorin und zum Autor}

Elisabeth von Salis, Psychoanalytikerin und Psychotherapeutin PSZ. Privatpraxis mit T. von Salis in St. Gallen und Zürich. Gruppenausbildung (A. Bauleo). Seit 1988 Gruppen-Ausbildnerin in Zürich. Familien- und Paartherapie, Teamsupervision, Weiter- und Fortbildung in psychiatrischen, kinderpsychiatrischen und anderen Institutionen. Mitglied AGOG, AIPCF, EFPP und AEPEA Schweizer Sektion. Buch-Publikation: Graf E. O. und von Salis E. (2003): Erfahrungen mit Gruppen. Theorie, Technik und Anwendungen der operativen Gruppe. Zürich: Seismo. Buchbeiträge in: von Salis T. (2019). Das Lernen und die Gruppe. Lit Verlag Münster.

Thomas von Salis, Kinder- und Jugendlichen Psychiatrie und Psychotherapie in Zürich, Dublin und St. Gallen. Psychoanalytische Ausbildung und Ausbildung in operativen Gruppen in Zürich. PSZ-Mitglied seit 1969. Berufspolitik: Präsident Schweizerische Gesellschaft für Kinderpsychiatrie und Sektion der Europäischen Union der Fachärzte. Diverse Kommissionen. AGOG (Arbeitsgemeinschaft für operative Gruppen), AIPCF, EFPP und AEPEA Schweizer Sektion. Das Lernen und die Gruppe (2019). Lit Verlag Münster. 